Quim. Nova, Vol. 35, No. 6, 1260-1263, 2012

\title{
SÍNTESE DA EPOXONE A PARTIR DE D-FRUTOSE. UM EXPERIMENTO DIDÁTICO EM LABORATÓRIO DE QUÍMICA ORGÂNICA COM FOCO NOS PRINCÍPIOS DA QUÍMICA VERDE
}

\author{
Tula Beck Bisol, Marcelo Volpatto Marques, Thaís Andreia Rossa, Maria da Graça Nascimento e Marcus Mandolesi Sá* \\ Departamento de Química, Universidade Federal de Santa Catarina, CP 476, 88040-900 Florianópolis - SC, Brasil
}

Recebido em 31/8/11; aceito em 20/12/11; publicado na web em 23/3/12

\begin{abstract}
SYNTHESIS OF EPOXONE FROM D-FRUCTOSE. A DIDACTIC EXPERIMENT FOR ORGANIC CHEMISTRY LABORATORY COURSES FOCUSING ON GREEN CHEMISTRY PRINCIPLES. In recent years, the introduction of the Green Chemistry concepts in undergraduate chemistry classes has been intensively pursued. In this regard, the two-step preparation of Epoxone (an organocatalyst developed by Shi \& col.) from commercial D-fructose, through ketalization of vicinal diols followed by oxidation of a sterically congested secondary alcohol, involves important topics in Organic Chemistry and employs inexpensive and nontoxic reagents. The reactions are easy to perform and the products from both steps are readily obtained as crystalline solids after simple procedures, thus facilitating their chemical characterization.
\end{abstract}

Keywords: epoxone; D-fructose; green chemistry.

\section{INTRODUÇÃO}

A crescente demanda pelo desenvolvimento de métodos sintéticos ambientalmente seguros e sustentáveis ${ }^{1-5}$ é uma tendência importante tanto no setor produtivo quanto na esfera acadêmica. Desta forma, o planejamento de aulas práticas nos cursos de Química deve abranger experimentos que estejam fundamentados nos princípios da Química Verde. ${ }^{6-9}$ Estes incluem, entre outros, a utilização de materiais de partida atóxicos e oriundos de fontes renováveis, além da escolha apropriada das condições reacionais e da readequação das etapas de tratamento e purificação com o objetivo de diminuir a geração de resíduos prejudiciais ao meio ambiente.

A oxidação de alcoóis a compostos carbonilados é um dos tópicos mais comuns em aulas experimentais de Química Orgânica. Os reagentes convencionais utilizados nestes experimentos normalmente são derivados de crômio, como $\mathrm{K}_{2} \mathrm{Cr}_{2} \mathrm{O}_{7}, \mathrm{CrO}_{3}$ e $\mathrm{PCC}$ (clorocromato de piridínio), que apesar de fornecerem bons rendimentos na oxidação de alcoóis a aldeídos ou cetonas, são substâncias tóxicas e geram resíduos que demandam cuidados especiais no seu descarte. Alternativamente, agentes oxidantes mais acessíveis e menos agressivos, como hipoclorito de sódio, TCICA (ácido tricloroisocianúrico) e Oxone $\left(2 \mathrm{KHSO}_{5} \times \mathrm{KHSO}_{4} \times \mathrm{K}_{2} \mathrm{SO}_{4}\right)$, têm sido introduzidos com sucesso em aulas experimentais. ${ }^{7,10-12}$

A organocatálise assimétrica vem apresentando um impacto significativo em síntese orgânica na última década, como ferramenta para a preparação de blocos de construção quirais e ingredientes farmacologicamente ativos. ${ }^{13-16}$ Além da reconhecida eficiência catalítica, organocatalisadores possuem diversas vantagens em relação aos complexos de metais de transição tradicionalmente empregados em síntese assimétrica, incluindo maior estabilidade, facilidade de acesso e menor custo. Portanto, este é um tópico que deve estar presente nos conteúdos programáticos de disciplinas de Química Orgânica. ${ }^{17}$

Um exemplo interessante de organocatalisador prático e de fácil acesso é a Epoxone (1), um derivado de carboidrato quiral desenvolvido por Shi e colaboradores, que promove a epoxidação assimétrica de olefinas di- e trissubstituídas na presença de Oxone em altos excessos enantioméricos. ${ }^{18}$ A preparação de $\mathbf{1}$ pode ser

*e-mail: marcus.sa@ufsc.br convenientemente realizada em duas etapas a partir da dicetalização da D-frutose (2) seguida de oxidação do álcool 3 ("Epoxol") para a cetona correspondente (Esquema 1). $\mathrm{O}$ fato de $\mathbf{2}$ ser um carboidrato abundante e oriundo de fontes renováveis, além de um produto alimentício amplamente disponível em estabelecimentos comerciais, torna atraente a sua utilização em um experimento de ensino.

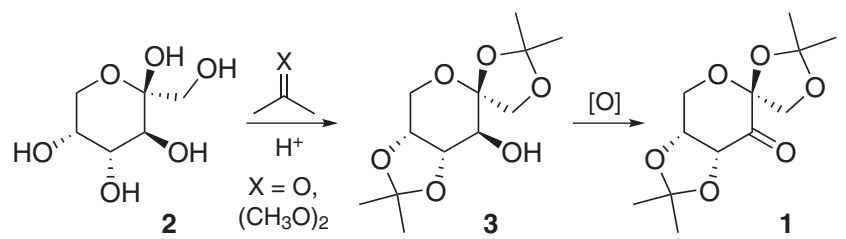

Esquema 1. Síntese da Epoxone (1) em duas etapas a partir de D-frutose (2)

A síntese de $\mathbf{1}$ a partir de D-frutose (2) foi adaptada para aulas experimentais de Química Orgânica e está descrita na literatura. ${ }^{19}$ Entretanto, a periculosidade associada ao uso de ácido perclórico $\left(\mathrm{HClO}_{4}\right)$ e de PCC nas etapas de dicetalização e oxidação, respectivamente, torna a manipulação destes reagentes imprópria aos alunos das disciplinas experimentais dos cursos de graduação. Além disso, as condições descritas incluem a necessidade de conduzir ambas as etapas reacionais sob atmosfera inerte, o que é inviável em laboratórios que não dispõem de infraestrutura adequada. Desta forma, decidiu-se revisar as metodologias previamente descritas na literatura para a preparação de $\mathbf{1}$ a partir de $\mathbf{2}^{20-25}$ e, de modo a desenvolver experimentos compatíveis com as condições existentes em nossos laboratórios de ensino, optou-se pela substituição de ácido perclórico e de PCC por reagentes alternativos.

Neste trabalho, é descrita a preparação da Epoxone (1) a partir de D-frutose (2) em duas etapas, utilizando condições simples, reagentes acessíveis e de baixa toxicidade. Os produtos de cada uma das duas etapas são sólidos cristalinos facilmente purificados por recristalização ou cromatografia em coluna e caracterizados por ponto de fusão. Além disso, outros métodos de análise, como ensaios em via úmida, espectroscopia de infravermelho (IV) e ressonância magnética nuclear de hidrogênio $\left(\mathrm{RMN}{ }^{1} \mathrm{H}\right)$, também podem ser utilizados. A seguir serão apresentados os resultados obtidos em aulas experimentais de Química Orgânica para cada uma das duas etapas da síntese. 


\section{PARTE EXPERIMENTAL}

\section{Preparação do Epoxol (3)}

A um balão de fundo redondo contendo uma suspensão de D-frutose (marca Doce Menor, 1,0 g, 5,6 mmol) em acetona (20 mL) foi adicionado, sob agitação vigorosa, $\mathrm{H}_{2} \mathrm{SO}_{4}$ conc. $(0,1 \mathrm{~mL})$ e a reação foi agitada a $25{ }^{\circ} \mathrm{C}$ até a dissolução completa dos sólidos (cerca de 1,5-2 h). Em seguida, resfriou-se a reação em banho de gelo e adicionou-se $\mathrm{NaOH} 2,5 \mathrm{M}(3,0 \mathrm{~mL})$. A mistura foi concentrada sob pressão reduzida e o resíduo obtido foi extraído com $\mathrm{CH}_{2} \mathrm{Cl}_{2}(20 \mathrm{~mL})$. A fase orgânica foi lavada com $\mathrm{H}_{2} \mathrm{O}(15 \mathrm{~mL})$ e brine $(15 \mathrm{~mL})$, seca com $\mathrm{Na}_{2} \mathrm{SO}_{4}$ anidro e concentrada sob pressão reduzida para fornecer um sólido amarelado com faixa de fusão $95-105^{\circ} \mathrm{C}$. A recristalização em hexano a quente ${ }^{26}$ produziu 3 na forma de agulhas $(0,3-0,6 \mathrm{~g}$, cerca de $20-40 \%$ de rendimento). Ponto de fusão médio: $116-118^{\circ} \mathrm{C}$ (Lit. ${ }^{20}$ $\left.117,5-118,0^{\circ} \mathrm{C}\right)$. IV (KBr): $v_{\max } 3460,2992,2944,2902,1389,1367$, $1248,1220,1117,1078 \mathrm{~cm}^{-1}$; RMN ${ }^{1} \mathrm{H}\left(200 \mathrm{MHz}, \mathrm{CDCl}_{3}\right): \delta 1,37$ (s, 3H), 1,44 (s, 3H), 1,51 (s, 3H), 1,53 (s, 3H), 2,02 (d, $J=7,5 \mathrm{~Hz}$, 1H), 3,65 (apt, $J=7,2 \mathrm{~Hz}, 1 \mathrm{H}), 3,95-4,25$ (m, 6H).

\section{Ensaio qualitativo com solução de bórax para detecção de dióis vicinais}

Uma solução de bórax [preparada a partir de uma solução aquosa diluída de bórax $\left(1 \% \mathrm{Na}_{2} \mathrm{~B}_{4} \mathrm{O}_{7}\right.$ em $\left.\mathrm{H}_{2} \mathrm{O}\right)$ e de algumas gotas de fenolftaleína ${ }^{27}$ em quantidade suficiente para a solução ficar rosa] foi adicionada a amostras de D-frutose (2), Epoxol (3) e Epoxone (1), fornecendo resultado positivo para a primeira (descoloração da solução rosa) e negativo para as duas últimas (manutenção da coloração rosa).

\section{Preparação da Epoxone (1)}

A um balão de fundo redondo contendo uma mistura de $3(0,52$ g, 2,0 mmol) em $\mathrm{CHCl}_{3}(2,0 \mathrm{~mL})$ e $\mathrm{H}_{2} \mathrm{O}(2,0 \mathrm{~mL})$ a $25^{\circ} \mathrm{C}$ foram adicionados, sob agitação magnética vigorosa, cloreto de benziltrietilamônio (BTEAC; 0,025 g, 0,1 mmol), $\mathrm{NaIO}_{4}(0,65 \mathrm{~g}, 3,0 \mathrm{mmol})$, $\mathrm{K}_{2} \mathrm{CO}_{3}(0,04 \mathrm{~g}, 0,3 \mathrm{mmol})$ e $\mathrm{RuCl}_{3} \cdot \mathrm{H}_{2} \mathrm{O}(0,01 \mathrm{~g}, 0,05 \mathrm{mmol})$. A reação foi agitada sob refluxo por $1,5 \mathrm{~h}$; em seguida, a fonte de aquecimento foi retirada para a adição cuidadosa, pelo topo do condensador, de isopropanol $(0,5 \mathrm{~mL})$ e a mistura resultante foi agitada por mais 0,5 h sob refluxo. Após resfriar o balão reacional, o sólido insolúvel foi removido por filtração em celite e lavado com $\mathrm{CH}_{2} \mathrm{Cl}_{2}(50 \mathrm{~mL})$. O filtrado orgânico foi então lavado com solução saturada de $\mathrm{Na}_{2} \mathrm{~S}_{2} \mathrm{O}_{3}$ $(20 \mathrm{~mL})$ e $\mathrm{H}_{2} \mathrm{O}(20 \mathrm{~mL})$, seco com $\mathrm{Na}_{2} \mathrm{SO}_{4}$ anidro e concentrado sob pressão reduzida para fornecer um sólido amarelo claro. A purificação por cromatografia em coluna $\left(R_{f}=0,59\right.$; eluente: hexano/EtOAc 1:1) utilizando um gradiente de hexano/EtOAc 4:1 até 1:1 forneceu 1 como um sólido branco cristalino (0,2-0,3 g, cerca de 40-60\% de rendimento). Ponto de fusão médio: $102-104{ }^{\circ} \mathrm{C}$ (Lit. ${ }^{28} 102-103{ }^{\circ} \mathrm{C}$ ). IV (KBr): $v_{\max } 2993,2940,1751,1457,1230,1097 \mathrm{~cm}^{-1} ; \mathrm{RMN}{ }^{1} \mathrm{H}$ $\left(200 \mathrm{MHz}, \mathrm{CDCl}_{3}\right): \delta 1,40(\mathrm{~s}, 6 \mathrm{H}), 1,46(\mathrm{~s}, 3 \mathrm{H}), 1,55$ (s, 3H), 3,99 $(\mathrm{d}, J=9,5 \mathrm{~Hz}, 1 \mathrm{H}), 4,12(\mathrm{~d}, J=13,5 \mathrm{~Hz}, 1 \mathrm{H}), 4,38(\mathrm{dd}, J=13,5$, $2,2 \mathrm{~Hz}, 1 \mathrm{H}), 4,50-4,55$ (m, 1H), 4,61 (d, $J=9,5 \mathrm{~Hz}, 1 \mathrm{H}), 4,73$ (d, $J$ $=5,5 \mathrm{~Hz}, 1 \mathrm{H})$.

\section{RESULTADOS E DISCUSSÃO}

Geralmente as aulas de Química Orgânica Experimental envolvem etapas de síntese, isolamento e caracterização de produtos que devem ser realizadas em períodos curtos (3-4 h). Portanto, muitas vezes são necessárias modificações e adaptações dos procedimentos citados na literatura. Os experimentos aqui descritos são modulares e podem ser desenvolvidos de diversas maneiras, sendo que o professor responsável pela disciplina opta pela aplicação de uma ou de duas etapas, dependendo das particularidades de cada curso e dos recursos disponíveis no laboratório. Por exemplo, a preparação e a recristalização do Epoxol (3) não demandam mais do que $4 \mathrm{~h}$ no total. Já a síntese e purificação da Epoxone (1) em duas etapas a partir de D-frutose (2) pode ser completada em 3 aulas de $3 \mathrm{~h}$ cada.

\section{Obtenção do Epoxol (3)}

A primeira etapa na preparação da Epoxone (1) é a dicetalização regiosseletiva de quatro das cinco hidroxilas da D-frutose (2) para a formação de $\mathbf{3}$ (Esquema 1). A diferença na reatividade das hidroxilas com formação de dois grupos acetonídeo distintos serve como um exemplo didático de seletividade relacionada à estereoquímica e pode ser um conteúdo explorado pelo professor. Os métodos descritos na literatura para dicetalização da D-frutose (2) envolvem a utilização de acetona (geralmente na presença de 2,2-dimetoxipropano) sob catálise ácida, com rendimentos na faixa de $33-53 \% .{ }^{19-23}$ Optou-se por uma combinação que inclui apenas acetona e ácido sulfúrico, ${ }^{20}$ devido à facilidade de aquisição e manipulação destes reagentes (Esquema 2). A reação é extremamente simples e, ao contrário do descrito na literatura para a utilização de ácido perclórico como catalisador, ${ }^{19,22}$ foi realizada sem a necessidade de atmosfera inerte, o que viabiliza a aplicação extensiva em aulas experimentais na maioria dos cursos no país.<smiles>OC[C@@]1(O)OC[C@@H](O)[C@H](O)[C@@H]1O</smiles>
$\underset{\substack{\mathrm{H}_{2} \mathrm{SO}_{4} \text { cat. } \\ 1,5-2 \text { h, t.a. }}}{\stackrel{\text { acetona }}{\longrightarrow}}$<smiles>CC1(C)O[C@H]2CO[C@]3(COC(C)(C)O3)C(O)[C@@H]2O1</smiles>

Esquema 2. Dicetalização da D-frutose (2)

O consumo de D-frutose (2) pôde ser acompanhado pela mudança de aspecto da mistura reacional, que passou inicialmente de turva para completamente límpida em cerca de 1,5 h. Após tratamento aquoso e retirada do solvente, o produto reacional bruto foi obtido como um sólido amarelo amorfo. Em seguida (ou na aula seguinte), o sólido foi recristalizado em hexano a quente, ${ }^{26}$ fornecendo o Epoxol (3) com alto grau de pureza em cerca de $20-40 \%$ de rendimento e com valores de ponto de fusão próximos aos descritos na literatura. ${ }^{19-23}$ Pela comparação dos valores de ponto de fusão dos produtos antes e depois da recristalização (ver Parte Experimental), ficou evidente a necessidade de incluir esta etapa de purificação no processo.

As reações de dicetalização foram reprodutíveis em escalas de 1,0 e 15 g. Os alunos confirmaram a obtenção de $\mathbf{3}$ pela medida do ponto de fusão da fração purificada e pela interpretação de seus espectros de IV (os dados espectrais obtidos estão apresentados na Parte Experimental e no Material Suplementar). Além disso, o ensaio com solução de bórax na presença de fenolftaleína ${ }^{27}$ (um teste simples para a detecção de dióis vicinais pela formação de complexos organoboratos cíclicos de caráter ácido), realizado tanto com o produto reacional bruto quanto com o material purificado, forneceu resultado negativo em ambos os casos como indicativo da ausência de D-frutose (2) (Esquema 3).

\section{Obtenção da Epoxone (1)}

A segunda etapa na preparação da Epoxone (1) é a oxidação da hidroxila livre do Epoxol (3). Devido à grande variedade de métodos conhecidos para a oxidação de alcoóis secundários às respectivas 


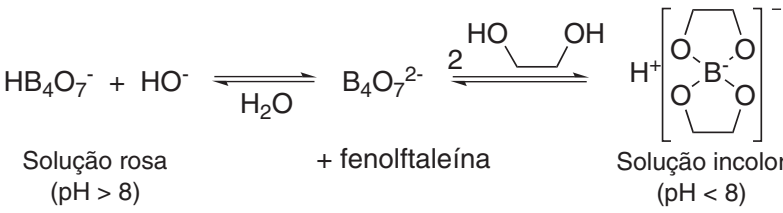

Esquema 3. Ensaio com solução de bórax para detecção de dióis vicinais

cetonas, este é um tópico importante a ser explorado pelo professor, podendo ser proposta aos alunos a elaboração de miniprojetos para aplicação de algumas destas metodologias visando a reação $\mathbf{3} \rightarrow \mathbf{1}$ fundamentada nos princípios da Química Verde. De fato, o grande impedimento estérico causado pelos grupos acetonídeo adjacentes ao centro carbinólico a ser oxidado limita o número de metodologias disponíveis para realizar esta transformação. A que utiliza PCC na presença de peneira molecular ${ }^{23}$ tem sido explorada com esta finalidade e foi aplicada em aulas experimentais de Química Orgânica, ${ }^{19}$ apesar da alta toxicidade dos reagentes envolvidos, dos resíduos gerados e da necessidade de utilização de atmosfera inerte.

Com o objetivo de sintetizar a Epoxone (1) sob condições reacionais mais brandas, ${ }^{24}$ optou-se por empregar $\mathrm{NaIO}_{4}$ e $\mathrm{RuCl}_{3} \mathrm{em}$ meio bifásico. ${ }^{25}$ Desta forma, a oxidação foi efetuada com tetróxido de rutênio $\left(\mathrm{RuO}_{4}\right){ }^{29}$ um forte agente oxidante gerado in situ pela combinação de $\mathrm{NaIO}_{4}$ com quantidades catalíticas de cloreto de rutênio hidratado e de BTEAC como catalisador de transferência de fase $^{25}$ (Esquema 4). Embora seja um reagente relativamente caro, o sal de rutênio(III) é empregado em pequenas quantidades (2-3 mol $\%$ ), o que permite sua utilização sem um aumento considerável dos custos. ${ }^{30}$ Deve-se ressaltar que, por se tratar de uma substância volátil halogenada, a necessidade do uso de clorofórmio é justificada pelo fato de ser, dentre os solventes comuns de laboratório, compatível com o sistema bifásico e as condições oxidativas do meio.
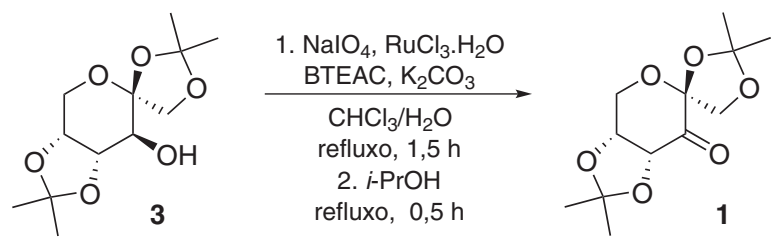

Esquema 4. Oxidação do Epoxol (3)

Após separação do catalisador, ${ }^{31,32}$ tratamento aquoso e eliminação do solvente, os produtos obtidos pelos grupos de alunos apresentaram leve coloração e foram, portanto, submetidos à purificação por cromatografia em coluna. Epoxone (1) foi obtida como um sólido branco com rendimento médio de 40-60\%. A obtenção de 1 foi confirmada por medida do ponto de fusão e por espectroscopia de IV (os dados espectrais obtidos estão apresentados na Parte Experimental e no Material Suplementar).

\section{CONCLUSÃO}

A síntese da Epoxone (1) em duas etapas a partir de D-frutose (2) é um experimento simples que abrange conceitos importantes, como Química Verde, organocatálise, estereoquímica, proteção de dióis vicinais e oxidação de alcoóis, e pode ser aplicado em qualquer laboratório de Química. O material de partida é um carboidrato amplamente disponível, além de barato, atóxico e oriundo de fontes renováveis. As condições envolvidas são brandas e o isolamento dos produtos sólidos é realizado de maneira simples, bem como sua caracterização por ponto de fusão. O experimento é modular e pode tanto ser realizado parcialmente em um único período de $3 \mathrm{~h}$ como ser planejado para a execução completa em torno de $10 \mathrm{~h}$ divididas em três períodos, integrando técnicas de montagem de reações e de isolamento e caracterização de produtos.

\section{MATERIAL SUPLEMENTAR}

Está disponível em http://quimicanova.sbq.org.br, na forma de arquivo PDF com acesso livre, e contém cópias dos espectros de IV e RMN ${ }^{1} \mathrm{H}$ dos compostos Epoxone (1) e Epoxol (3).

\section{AGRADECIMENTOS}

Aos alunos dos cursos de Química e de Farmácia que participaram das aulas experimentais, aos funcionários da Central de Análises e do Departamento de Química da UFSC, ao INCT-Catálise e às agências de fomento CAPES, CNPq e FAPESC pelas bolsas de estudo e pelo apoio financeiro.

\section{REFERÊNCIAS E NOTAS}

1. Tucker, J. L.; Org. Process Res. Dev. 2010, 14, 328.

2. Anastas, P. T.; Tetrahedron 2010, 66, 1026.

3. Hudlicky. T.; Chem. Rev. 2011, 111, 3995.

4. Sheldon, R. A.; Green Chem. 2007, 9, 1273.

5. Dallinger, D.; Kappe, C. O.; Chem. Rev. 2007, 107, 2563.

6. Braun, B.; Charney, R.; Clarens, A.; Farrugia, J.; Kitchens, C.; Lisowski, C.; Naistat, D.; O'Neil, A.; J. Chem. Educ. 2006, 83, 1126.

7. Santos, A. P. B.; Gonçalves, I. R. C.; Pais, K. C.; Martinez, S. T.; Lachter, E. R.; Pinto, A. C.; Quim. Nova 2009, 32, 1667.

8. Navarro, M.; Sena, V. L. M.; Srivastava, R. M.; Navarro, D. M. A. F.; Quim. Nova 2005, 28, 1111.

9. Merat, L. M. O. C.; Gil, R. A. S. S.; Quim. Nova 2003, 26, 779.

10. Lang, P. T.; Harned, A. M.; Wissinger, J. E.; J. Chem. Educ. 2011, 88, 652.

11. Mohrig, J. R.; Hammond, C. N.; Morrill, T. C.; Neckers, D. C.; Experimental Organic Chemistry, W. H. Freeman and Company: New York, 1998.

12. Apostila do DQ-UFSC, disponível em www.qmc.ufsc.br/organica, acessada em Fevereiro 2012.

13. Dondoni, A.; Massi, A.; Angew. Chem., Int. Ed. 2008, 47, 4638.

14. MacMillan, D. W. C.; Nature 2008, 455, 304.

15. Bruckmann, A.; Krebs, A.; Bolm, C.; Green Chem. 2008, 10, 1131.

16. Pellissier, H.; Tetrahedron 2007, 63, 9267.

17. Wade, E. O.; Walsh, K. E.; J. Chem. Educ. 2011, 88, 1152.

18. Wong, O. A.; Shi, Y.; Chem. Rev. 2008, 108, 3958.

19. Burke, A.; Dillon, P.; Martin, K.; Hanks, T. W.; J. Chem. Educ. 2000, 77, 271.

20. Kang, J.; Lim, G. J.; Yoon, S. K.; Kim, M. Y.; J. Org. Chem. 1995, 60, 564.

21. Ager, D. J.; Anderson, K.; Oblinger, E.; Shi, Y.; VanderRoest, J.; Org. Process Res. Dev. 2007, 11, 44.

22. Wang, Z.-X.; Tu, Y.; Frohn, M.; Zhang, J.-R.; Shi, Y.; J. Am. Chem. Soc. 1997, 119, 11224.

23. Tu, Y.; Frohn, M.; Wang, Z.-X.; Shi, Y.; Org. Synth. 2003, 80, 1.

24. Gonsalvi, L.; Arends, I. W. C. E.; Sheldon, R. A.; Org. Lett. 2002, 4, 1659.

25. Mio, S.; Kumagawa, Y.; Sugai, S.; Tetrahedron 1991, 47, 2133.

26. Cuidado! Hexano é uma substância inflamável (ponto de fulgor = $-35^{\circ} \mathrm{C}$ ) e sua utilização sob aquecimento deve estar em consonância com as normas de segurança recomendadas (www.higieneocupacional. com.br/download/hexano-petrobras.pdf, acessada em Fevereiro 2012).

27. Shriner, R. L.; Hermann, C. K. F.; Morrill, T. C.; Curtin, D. Y.; Fuson, R. C.; The Systematic Identification of Organic Compounds, $8^{\text {th }}$ ed., John Wiley \& Sons: Hoboken, 2004, p. 308; Vogel, A. I.; Furnis, B. S.; 
Hannaford, A. J.; Smith, P. W. G.; Tatchell, A. R.; Vogel's Textbook of Practical Organic Chemistry, $5^{\text {th }}$ ed., Longman Scientific \& Technical: Essex, 1989, p. 1224.

28. Zhang, Z.; Tang, J.; Wang, X.; Shi, H.; J. Mol.Catal. A: Chem. 2008, 285, 68.

29. Rup, S.; Sindt, M.; Oget, N.; Tetrahedron Lett. 2010, 51, 3123.

30. $1,0 \mathrm{~g}$ de $\mathrm{RuCl}_{3} \cdot \mathrm{H}_{2} \mathrm{O}$ (número do registro CAS 14898-67-0) custa $€ 37,90$ (ACROS - número do produto 195480010; fonte: www.acros.com, acessada em Fevereiro 2012). Com $1,0 \mathrm{~g}$ de $\mathrm{RuCl}_{3} \cdot \mathrm{H}_{2} \mathrm{O}$, cerca de 100 equipes poderiam realizar a oxidação de $0,5 \mathrm{~g}$ de Epoxol (2).
31. Para evitar que o material de laboratório utilizado nos experimentos de oxidação com sal de rutênio fique impregnado com traços do catalisador, é importante tratar toda a vidraria com solução aquosa de hipoclorito de sódio (água sanitária 3\%) antes da lavagem convencional com água e detergente.

32. Resíduos contendo metais no estado sólido ou em solução devem ser separados e identificados para tratamento e disposição final. 